proposed in 1976 (Offen ML, et al. J Neurol Neurosurg Psychiatry 1976 Sep;39(9):829-34). Hypothalamic hamartoma is the most likely cause when dacrystic seizures (DC) are accompanied by gelastic epilepsy. When DS occur alone, the lesion is commonly in the temporal lobe cortex. DS are refractory to treatment with AEDs and frequently require surgery for removal of a structural lesion. Whereas involuntary laughter is an accepted expression of epilepsy, especially as a symptom of hypothalamic hamartoma, involuntary crying is a relatively rare form of epilepsy. The current article adds to the sparse literature suggesting that DS are symptomatic of structural brain lesions, and demonstrates that patients with DS without gelastic epilepsy frequently present with mesial temporal sclerosis. Symptoms leading to the diagnosis of a hypothalamic-pituitary or temporal lobe lesion are usually neurological, including increased intracranial pressure and seizures. Endocrine symptoms (changes in weight, height, puberty, or diabetes insipidus) and their occurrence prior to the onset of neurologic symptoms may help to diagnose the hypothalamic-pituitary lesions earlier than the appearance of gelastic seizures (Taylor M, et al. J Pediatr 2012 Nov;161(5):855-863.e3; see Pediatr Neurol Briefs 2012 Dec;26(12):95-96).

\title{
UTILITY OF SHORT-TERM VIDEO-EEG MONITORING
}

Researchers at Monash University Medical Center, Melbourne, Australia evaluated the yield and clinical utility of outpatient, short-term video-EEG monitoring (OVEM) as a diagnostic tool in routine clinical practice. Of a total of 175 patients with records examined retrospectively, 111 were female and 64 male, with an age-range of 1687 years (mean 36 years). Mean length of recording was $3.8 \mathrm{hrs}$ (range 1-6.8 hrs). Pretest frequencies of clinical events were $<1$ per week (30.1\%), 2-6 per week $(48.7 \%)$, and $>7$ per week $(21.2 \%)$. Focal slowing occurred in 24 recordings $(13.7 \%)$ and background and generalized slowing in $18(10.3 \%)$. Interictal epileptiform discharges (IED) were focal in $15(8.6 \%)$ and generalized in the same frequency. Epileptic seizures were captured in 12 patients (6.9\%). Psychogenic nonepileptic seizures (PNES) occurred in 65 (37.1\%) patients. The diagnostic yield for PNES was 37.1\%, for IED 17.2\%, and for epileptic seizures $6.9 \%$. Before OVEM, a provisional diagnosis of epilepsy was made in $136(77.7 \%)$ patients; after OVEM, the diagnosis of epilepsy was changed to PNES in $28.6 \%$, and from PNES to epilepsy in 2.3\%. OVEM has a higher yield for PNES than epileptic seizures and IED. The yield of PNES was $>5$ times that of epileptic seizures, and diagnosis was changed from epilepsy to PNES in $>$ one-fourth of patients. (Seneviratne U, Rahman Z, et al. The yield and clinical utility of outpatient short-term video-electroencephalographic monitoring: A five-year retrospective study. Epilepsy Behav 2012 Nov;25(3):303-6). (Respond: Dr Udaya Seneviratne, Dept. of Neuroscience, Monash Medical Centre, Australia. E-mail: udaya.seneviratne@monash.edu).

COMMENT. Outpatient short-term VEM changes the pre-test diagnosis in $30.9 \%$ of patients. It is a useful diagnostic test for PNES and has a higher yield for PNES than epilepsy. OVEM is relatively cheaper than inpatient long-term VEM, but the shorter recording duration may miss some patients with epileptic seizures. OVEM is considered in the diagnostic work-up of suspected PNES prior to an inpatient long-term VEM. 